DOI: http://dx.doi.org/10.12957/demetra.2014.9461

\title{
Alterações na identidade social do obeso: do estigma ao fat pride
}

\section{Changes in social identity of the obese: from stigma to the fatprice}

Alden dos Santos Neves'

André Luís de Oliveira Mendonça²

1 Programa de Pós-graduação em Alimentação, Nutrição e Saúde, Instituto de Nutrição. Universidade do Estado do Rio de Janeiro. Rio de Janeiro-RJ, Brasil.

${ }^{2}$ Instituto de Medicina Social. Universidade do Estado do Rio de Janeiro. Rio de Janeiro-RJ, Brasil.

Correspondência /Correspondence E-mail: aldensn@gmail.com

\section{Resumo}

O fenômeno da transição nutricional trouxe como principal consequência uma alta prevalência de sobrepeso / obesidade na população mundial. Além dos reflexos para a saúde, a obesidade traz alterações sociais importantes aos portadores desta condição. A despeito do crescimento epidêmico da obesidade, pode-se perceber o surgimento de uma sociedade lipofóbica, que transforma os obesos em portadores de um estigma, pela associação da obesidade com uma série de valores morais negativos. O surgimento do movimento fat pride caracteriza uma reação do obeso ao estigma da obesidade, e a medicalização da obesidade aponta para a cirurgia bariátrica como tecnologia médica capaz de transformar a condição do indivíduo obeso. $\mathrm{O}$ presente artigo justifica-se pela necessidade de compreender as transformações na identidade social do obeso a partir de estudos de autores que abordam o problema da obesidade sob o referencial da Saúde Coletiva, tendo como objetivo geral compreender as transformações da identidade social do obeso, e como objetivos específicos: analisar a obesidade enquanto um estigma social e descrever brevemente a medicalização da obesidade e os fenômenos envolvidos no surgimento do fat pride. O surgimento deste movimento no Brasil difere do movimento original americano, embasando-se no apelo midiático e tendendo a criar um movimento comercial.

Palavras-chaves: Obesidade. Transição Nutricional. Estigma Social. Medicalização. 


\section{Abstract}

The phenomenon of nutritional transition brought as major consequence high prevalence of overweight / obesity in the world population. Beyond the reflections on health, obesity brings important social changes to those who suffer of this condition. Despite the growing epidemic of obesity, we can see the emergence of a lipophobic model of society, that puts on obese a stigma by association of obesity with a number of negative moral values. The arise of the fat pride movement features a reaction of the stigma of obesity, and the medicalization of obesity points to bariatric surgery as medical technology able to change the condition of the obese. This paper aims to justify the need to understand the changes in the social identity of the obese from studies of authors who address obesity under the framework of the Public Health. The overall objective is to understand the transformations of the social identity of the obese, and specific objectives: examine obesity as a social stigma, and briefly describe the medicalization of obesity and the phenomena involved in the emergence of fat pride. The emergence of this movement in Brazil differs from the original American movement, based on the media appeal, and tends to create a commercial movement.

Key words: Obesity. Nutritional Transition. Stigma. Medicalization.

\section{Introdução}

A obesidade é definida como a condição de acúmulo de peso corporal em forma de gordura, que pode levar ao surgimento de comorbidades de grande impacto para a saúde do indivíduo.1

O Brasil, seguindo a tendência mundial, vem vivenciando nos últimos 40 anos o aumento dos níveis de obesidade na população, resultante da conjugação de diversos fatores. Este fenômeno, conhecido como transição nutricional, surge como consequência de alterações no modo de vida da população em geral, principalmente como resultado da industrialização, urbanização, sedentarismo e saída da mulher para o mercado de trabalho. 2

Atualmente, 50,1\% da população brasileira encontram-se em algum grau de sobrepeso, e cerca de $16 \%$ da população adulta encontra-se obesa.3 Tais mudanças no padrão nutricional da população trazem consequências diretas para a saúde dos indivíduos, com reflexos para o estado 
de saúde da população como um todo. Outro aspecto relevante é como esse aumento epidêmico nos níveis de obesidade gera alterações sociais importantes, que impactam na vida diária dos portadores da condição.

A despeito do crescimento dos níveis de obesidade, percebe-se hoje o surgimento de uma sociedade lipofóbica, que estabelece valores associados ao culto do corpo, e que são realçados pela mídia, tornando os obesos indivíduos marcados socialmente, como portadores de um estigma.4 A condição social do obeso é marcada por discriminações de diversos matizes, e associa-se à obesidade uma gama de conceitos e valores negativos, criando uma situação de menos valor e depreciação.5

Para tornarem-se livres dessa condição social, os indivíduos obesos submetem-se a uma série de terapias, nem sempre com sucesso. Dentre as terapias utilizadas para o tratamento da obesidade, a cirurgia bariátrica surge como uma alternativa mais recente, e aparentemente mais eficiente, para a reversão do quadro de obesidade, e desponta como um ícone da medicalização da obesidade na atualidade.

No entanto, para a grande massa que ainda sofre com a condição da obesidade, um movimento começa a despontar como uma via alternativa ao estigma da obesidade. O fat pride apresentase como um fenômeno que começa a despontar no Brasil, com uma vertente mais midiática, se comparado ao movimento original norte-americano, mais focado em questões sociais, e que poderia tornar a condição obesa um pouco mais tolerável, fazendo a obesidade ser mais aceita na sociedade atual.

Este artigo se justifica pela necessidade de compreender as questões sociais relativas à obesidade, a partir de aportes teóricos das ciências sociais e humanas do campo da Saúde Coletiva, tomando por base estudos de autores que tratam do tema. Tem como objetivo geral compreender as transformações da identidade social do obeso, e como objetivos específicos: analisar a obesidade enquanto um estigma social, descrevendo brevemente a medicalização da obesidade e os fenômenos envolvidos no surgimento do fat pride. O trabalho abordará o fenômeno da transição nutricional e o crescimento da obesidade, o estigma social da obesidade, o papel da mídia e da sociedade no problema em questão, o processo de medicalização da obesidade e o surgimento do movimento fat pride, apontando as diferenças percebidas no movimento original americano quando comparado ao movimento brasileiro.

\section{Obesidade e transição nutricional}

A sociedade brasileira, seguindo uma tendência mundial instalada nos países desenvolvidos há aproximadamente meio século, apresentou nos últimos 40 anos um acelerado processo de urbanização, que trouxe consequências diretas à saúde de sua população. Tal processo mudou 
radicalmente as formas tradicionais de vida das populações que migraram para as cidades, com novas formas de trabalho e alimentação, além de propiciar maior acesso a serviços básicos de saúde. Como consequência de tais alterações, ocorreram mudanças na composição demográfica da população, permitindo aumento na expectativa de vida, diminuição da taxa de natalidade e alterações nos padrões nutricionais. Entretanto, tais modificações são acompanhadas de alterações epidemiológicas importantes, como o crescimento de doenças crônicas não transmissíveis, especialmente a obesidade, diabetes melito tipo 2, hipertensão, dentre outras, que apresentam correlações causais com alterações no padrão energético da dieta e diminuição da atividade física, relacionados ao modo urbano de vida. ${ }^{2}$

Tais mudanças se refletem de modo negativo, especialmente se forem consideradas as importantes transformações em gasto energético associado ao trabalho e alterações no consumo alimentar. O processo de industrialização do país fez com que houvesse modificações importantes no quadro distributivo da mão de obra, como a redução das ocupações nos setores primários da economia em toda a população, acompanhada de aumento nos setores do comércio e prestação de serviços. ${ }^{6}$

Em relação a essas mudanças no modo de trabalho, percebe-se que, com a migração do meio rural para o meio urbano, houve forte tendência de redução do gasto calórico diário rotineiro e laboral, pela maior utilização de transportes coletivos e individuais em substituição a caminhadas e uso de bicicletas, e pelo trabalho em processos automatizados ou semiautomatizados, em substituição ao trabalho braçal. ${ }^{7}$

Alterações significativas no padrão alimentar brasileiro também vêm ocorrendo paralelamente a este fenômeno de urbanização, com a mudança de um padrão tradicional caracterizado pela presença de raízes, frutas e grãos, por um padrão globalizado de alimentação, caracterizado pelo aumento significativo do consumo de sódio, açúcares simples e gorduras totais e saturadas, num modelo definido como "dieta ocidental". 8

Essas importantes mudanças relacionadas à adoção do novo estilo de alimentação vêm causando aumento significativo da obesidade, especialmente a obesidade infantil, associado a um decréscimo significativo nas taxas de desnutrição, caracterizando a transição nutricional brasileira. ${ }^{9}$

Como principal consequência da transição nutricional brasileira, pode-se perceber o aumento significativo na prevalência de sobrepeso e obesidade na população. De acordo com a última Pesquisa de Orçamentos Familiares (POF), de 2008-2009, 50,1\% da população brasileira adulta apresenta peso acima do ideal (sobrepeso), sendo que $16 \%$ da população estão obesos. ${ }^{3}$ 


\section{0 estigma da obesidade}

De acordo com Goffman, ${ }^{10}$ é uma característica das sociedades definir categorias mais ou menos rígidas acerca dos atributos considerados normais, naturais ou comuns do ser humano, ou a criação de uma identidade social. O indivíduo portador de características que não o classifiquem como um ser humano normal, natural ou comum é considerado portador de um estigma. $\mathrm{O}$ indivíduo estigmatizado é, então, aquele cuja identidade social inclui qualquer atributo que frustre as expectativas de normalidade observadas pela sociedade em questão. O estigma, então, passa a ser uma forma de classificação social, que passa pelo corpo e pelas relações sociais dos sujeitos.

Podemos encontrar referências à obesidade como um estigma desde a Bíblia. ${ }^{11} \mathrm{~A}$ estética do "corpo magro" surgiu no fim do século XIX e ganhou força durante todo o século XX, 4,5,12-15 intensificando-se nas duas últimas décadas do século. ${ }^{16} \mathrm{~A}$ obesidade pode ser vista como uma condição favorável quando a magreza está relacionada a padrões de doença, fome e privação. Com a mudança nos padrões de ingestão calórica na população, fruto de mais acesso à produção de alimentos, condição característica das sociedades industrializadas contemporâneas, o fenômeno da lipofobia se instala e se estabelece ao longo do século XX.${ }^{17}$ Segundo Eknoyan, ${ }^{13}$ no fim do século XIX, o indivíduo extremamente obeso era visto pela sociedade norte-americana como moralmente repreensível e, de acordo com Boero, ${ }^{12}$ no início do século XX surgiu a estética do "naturalmente magro" que, para as mulheres brancas, era vista como uma condição sine qua non para torná-las aptas ao casamento. A magreza, nos últimos 50 anos, passou a ser considerada de modo mais acentuado como sinônimo de saúde, distinção social e autocontrole. ${ }^{17}$

Neste início do século XXI, vivemos um momento de culto social ao corpo perfeito, com a hegemonia da tríade "beleza, juventude e saúde", em um movimento sustentado pelo meio científico e pela mídia. Os indivíduos passam a ser considerados responsáveis pelo desenvolvimento dos valores fundamentais desta tríade em seu corpo, e são culpabilizados pela sociedade caso não consigam imprimir em seu corpo esses valores. ${ }^{5}$

O corpo passa a ser um instrumento da construção da identidade, muito influenciado pelas imposições sociais (que vão da educação à mídia), sendo exposto às mais diversas formas de controle para atingir o modelo que seria a consonância entre saúde e beleza, através de exercício, consumo de drogas e práticas alimentares desgastantes. ${ }^{14,15}$ Os ideais culturais norte-americanos de valorização do esforço, trabalho duro e autocontrole apontam a obesidade como uma condição de preguiça no imaginário popular. 
Em relação a este modelo de corpo, o corpo obeso seria a medida exata dos valores que são diretamente opostos aos valores em voga na sociedade. O modelo de saúde perfeita, baseada em corpos perfeitos, magros, sem nenhuma imperfeição, reforça o preconceito social contra a obesidade. ${ }^{5,4}$ A excessiva valorização do corpo magro transforma a gordura corporal em algo indesejável, um símbolo de falência. O portador da condição de obesidade, por não se adequar aos padrões vigentes de beleza e conformidade, passa a carregar este estigma. ${ }^{4}$

Existe uma opressão contra o corpo obeso, a partir do momento em que o corpo magro passa a ser uma condição prioritária para permitir uma vida social plena. Existe uma pressão cultural para emagrecer e controlar o peso, condição que reforça o sentimento lipofóbico da atualidade. E é exatamente esta supervalorização do corpo magro, "adestrado", que associa à gordura um símbolo de falência moral, pela falta de controle sobre o corpo, e o indivíduo obeso tem seu estigma reforçado.

Ao correlacionarmos os principais valores morais relacionados à obesidade à ideia defendida por Sontag ${ }^{18}$ da doença como metáfora ou um símbolo, pode-se perceber que a obesidade vem alcançando na estrutura da sociedade um papel semelhante ao descrito pela autora para o câncer. As duas condições estariam relacionadas ao excesso, ao descontrole (emocional ou físico), por parte daqueles que lhe são os portadores.

De acordo com as ideias da mesma autora, a recuperação de uma doença depende do uso enérgico da vontade para criar as condições de recuperação, sendo a cura a efetivação da capacidade do próprio doente de criar ou utilizar os próprios recursos para a superação das causas daquela condição. Os obesos, como parte do estigma que carregam, são definidos como indivíduos incapazes de controlar sua vontade em relação à comida, passando a obesidade a ser vista como uma situação de fraqueza e pouca tenacidade moral, comparável com a situação de um adicto de algum tipo de droga. ${ }^{15}$ Tal conceito pode ser considerado como estigmatizante ou de culpabilização do portador da condição. Como diz Sontag: "Nada é mais punitivo do que atribuir um significado a uma doença quando esse significado é... moralista”. ${ }^{18}$

De acordo com o artigo de Rosa \& Campos, ${ }^{19}$ a chamada “condição obesa” gera grande impacto na subjetividade, com muito sofrimento e impedimentos sociais, especialmente como a sociedade (e também os indivíduos portadores de obesidade) lidam com as consequências da chamada "condição obesa”. Esta forma de representação social individualiza a questão da obesidade, colocando-a sob a estrita responsabilidade do indivíduo obeso, centralizando o problema no controle do seu próprio corpo e vontades. ${ }^{20}$ Num modelo de sociedade que cultua a imagem pessoal, sendo esta cada vez mais valorizada, os obesos não se enquadram nesse perfil pela impossibilidade de construir uma imagem agradável aos seus olhos e aos dos outros. ${ }^{21}$ 
Utilizando as ideias da mesma autora, vive-se atualmente um modelo de sociedade que aprecia e valoriza a rapidez e a leveza; nesse contexto, um corpo obeso evoca valores associados à imobilidade, improdutividade. Esta condição parece chocar-se com o ritmo frenético imposto pelo mercado de trabalho, que associado à possibilidade de afastamento das atividades laborais provocado pelas comorbidades presentes na condição obesa, promove uma discriminação do obeso pelo mercado de trabalho.

Como exemplo desta afirmativa, recentemente, nos Estados Unidos, os indivíduos severamente obesos ou com índice de massa corporal (IMC) acima de $40 \mathrm{~kg} / \mathrm{m}^{2}$ foram considerados como aptos a solicitar proteção através de estatutos legais que coíbem a discriminação contra deficientes pelo mercado de trabalho. ${ }^{11}$

\section{Mídia, sociedade e obesidade}

O modelo imposto pelo novo padrão cultural deste início de século vem sendo legitimado pelo discurso biomédico e produz um modelo no qual os indivíduos devem se encaixar. Esse conjunto de medidas forma um padrão de beleza e saúde que se espalha pela sociedade através das revistas, jornais e televisão. O modo biomédico de representar o corpo prioriza questões quantitativas, utilizando apenas parâmetros biológicos, desconsiderando e não valorizando as questões subjetivas, como aspectos culturais, sociais etc. Entretanto, considerar apenas a dimensão biológica, sem considerar as questões culturais e sociais, é parte de um modelo reducionista. ${ }^{5}$ Promover o estudo da obesidade a partir de contextualizações socioculturais proporciona o surgimento de uma série de questões relacionadas à construção cultural do corpo que ultrapassa esse modelo reducionista. ${ }^{11}$

Os modelos ideais de corpo são alardeados pela mídia, e contribuem fortemente para a deformação da autoimagem desenvolvida pelos indivíduos obesos. ${ }^{21} \mathrm{~A}$ mídia, ao expor constantemente a estética do corpo disciplinado, esculpido, construído à custa de esforço e domínio da vontade própria, negligencia os indivíduos à margem desta condição, contribuindo para reforçar a ideia do obeso como desprovido de vontade, de controle próprio. ${ }^{5,21}$ Historicamente, Gilman, ${ }^{11}$ abordando o problema da obesidade no povo judeu, relata que a obesidade era considerada um sinal de falta de disciplina, passível de punição, por não ser condizente com o controle necessário a um homem íntegro e digno. A relação histórica entre obesidade e valores morais depreciativos é percebida de muito longa data, como pode se perceber, por exemplo, na relação entre o jejum e corpos magros e espiritualidade, retratados na cultura da Idade Média, e a aversão dos espartanos pelas pessoas obesas. ${ }^{13}$ 
Valores depreciativos, como falta de vontade, preguiça, falta de saúde, falta de atração sexual, dentre outros, ainda estão fortemente relacionados à obesidade. ${ }^{22,23}$ As pessoas obesas são retratadas na mídia através de sub-representações e estereótipos. Por exemplo, pessoas com sobrepeso, especialmente personagens femininas, são retratadas como objetos de humor e são menos retratadas nos relacionamentos românticos, ao contrário das magras.

Recentemente, as reações populares contra uma série de TV que mostrou um beijo entre um casal romântico formado por dois obesos mórbidos trouxeram à tona questões relacionadas à sexualidade, e como a obesidade é vista por grande parte da sociedade como um impedimento para a experiência sexual plena. ${ }^{23}$ Ao mesmo tempo, os meios de comunicação de massa associam a felicidade à figura esbelta; as propagandas sobre produtos e serviços para emagrecer realçam a preocupação com as consequências individuais da obesidade, centralizando nos indivíduos a responsabilidade. ${ }^{20}$

Estas representações midiáticas da obesidade reforçam a associação entre a gordura corporal e o estigma da indolência, incapacidade (laborativa, física etc.) e até mesmo a exposição (voluntária, por considerar a existência da obesidade uma falta de controle) a uma série de riscos físicos, como as comorbidades da obesidade. O corpo esbelto é exposto como um sinal de controle, um indício de mente sã, enquanto o corpo obeso está relacionado ao descontrole e compulsão, visto quase como que ameaçado a uma forma contemporânea de loucura. ${ }^{14}$

\section{A medicalização da obesidade}

pode-se definir como medicalização o processo pelo qual situações originalmente não médicas passam a ser tratados como problemas ou situações médicas. ${ }^{24}$ Segundo Rasmussen, ${ }^{15}$ a obesidade, ao longo do século XX, transitou entre o estigma e a medicalização.

As explicações biomédicas para a obesidade surgiram com bastante evidência nos Estados Unidos no período entre as grandes guerras mundiais, com o advento da endocrinologia e as formas de tratamento médico baseadas em hormônios. ${ }^{15}$ A medicalização da obesidade começou a ocorrer de modo mais evidenciado a partir do fim da $2^{\text {a }}$ Grande Guerra. ${ }^{12}$

O termo "epidemia" de obesidade foi utilizado inicialmente pela mídia, revistas médicas e pela política de saúde pública para descrever o aumento da sua prevalência, fato percebido inicialmente nos Estados Unidos. O uso desse termo tem se tornado cada vez mais frequente para descrever o crescimento dos níveis de obesidade nos Estados Unidos, e pode-se evidenciar o papel da mídia na construção do conceito de epidemia na sociedade, e no modo como a população percebe o crescimento do sobrepeso/obesidade, baseando-se em discursos sobre peso, moralidade, ciência etc. ${ }^{12}$ Desta maneira, o discurso da epidemia passou a fornecer formas de oferecer explicações individuais para questões sociais. ${ }^{21}$ Além da "epidemia", outros termos metafóricos comumente 
utilizados por obesos para descrever suas tentativas de emagrecer são a "guerra contra a obesidade", sendo esta guerra constituída de várias "lutas" ou "batalhas" contra um inimigo perverso e resistente: a gordura corporal. ${ }^{21}$

De acordo com o paradigma biomédico, as doenças são como objetos concretos, sem significados social, cultural, emocional ou psíquico, e fazem com que a visão do médico, enquanto profissional de saúde, seja muito diferente da visão dos pacientes sobre o mesmo processo. ${ }^{25}$

No caso da obesidade, doença com severas implicações sociais, psicológicas, emocionais etc., a visão dos demais profissionais de saúde que lidam com a questão sobre as necessidades do paciente pode estar descontextualizada, por ser severamente influenciada pelo modelo reducionista posto em voga pelo paradigma biomédico. A despeito dos conjuntos de definições médicas sobre obesidade, a influência do contexto cultural sobre os portadores de obesidade é variável, mudando de uma cultura para outra com o passar do tempo. ${ }^{11}$

No entantanto, ao se considerar a obesidade como doença, conforme definida pela literatura técnico-científica, faz-se necessário buscar um tratamento efetivo para a patologia, e assim, a escolha da cirurgia se justifica. A crença no poder das tecnologias médicas intervindo no corpo, transformando-o e adequando-o aos padrões considerados normais, legitima a escolha pela cirurgia como caminho adequado para corrigir os "desvios" do corpo obeso, permitindo sua normatização. ${ }^{21}$

As técnicas e tecnologias médicas de transformação corporal acenam com a promessa de superação das barreiras e fronteiras da ordem social, ${ }^{14}$ possibilitando ao indivíduo obeso maior controle sobre seu corpo. As mudanças no tratamento da obesidade podem ser percebidas nas mudanças de denominação das sociedades que se devotam ao tratamento desta condição. Pode-se citar, como exemplo, nos Estados Unidos, a National Obesity Society, que foi renomeada em 1950 para National Glandular Society, e depois, para American College of Endocrinology and Nutrition; a partir de 1961, para American Society of Bariatrics, e finalmente, para American Association of Bariatrics Surgery, no fim dos anos 90 do século XX, demonstrando a entrada da cirurgia no tratamento da obesidade. Esta mudança reflete de forma clara as mudanças no conceito de tratamento da condição. ${ }^{13}$

Considerando que os portadores de formas graves de obesidade, e de acordo com os protocolos clínicos, elegíveis para esse tipo de procedimento, correm maior risco de morte e apresentam frequentemente histórias clínicas de insucessos recorrentes com os modelos de terapia antiobesidade convencionais (dietas, medicamentos, exercícios físicos e até mesmo psicoterapia), a cirurgia bariátrica passou a ser idealizada como forma de se livrar definitivamente desse risco físico e, consequentemente, de conseguir maior aceitação no meio social. Com os avanços das técnicas utilizadas, os riscos e inadequações geradas pelo tratamento cirúrgico passaram a ser considerados menos importantes pelos portadores de obesidade grave, frente à miríade de possibilidades de novas formas de vida. ${ }^{19,26}$ 


\section{0 movimento fat pride}

O movimento fat pride surgiu por volta de meados da década de 60 do século passado, nos Estados Unidos, com manifestações públicas contra discriminação e pela aceitação social da obesidade. Uma das organizações que sobrevive até a atualidade, e pode ser considerada um grande expoente deste movimento é a National Association for Advance of Fat Acceptance (NAAFA), fundada em 1969, e que apresenta como objetivos principais a construção de uma sociedade na qual os indivíduos "de todos os tamanhos" possam ser aceitos com igualdade e dignidade. ${ }^{27}$

A NAAFA foi fundada por William Fabrey, marido de uma obesa, cansado de perceber a discriminação social sofrida diariamente por sua esposa, e foi originalmente denominada de National Association to Aid Fat Americans. Atualmente, a NAAFA desenvolve uma série de medidas baseadas em apoio, educação pública ou suporte jurídico contra a discriminação de pessoas obesas, como ações contra empresas aéreas que cobram duas passagens aéreas de pessoas obesas para ocupar apenas um assento. ${ }^{22,27}$

Tomando como base os estudos de Goffman ${ }^{10}$ sobre estigma, pode-se considerar o surgimento do fat pride como uma estratégia utilizada para o enfrentamento do estigma, como uma possibilidade de reduzir o peso social do estigma e tornar mais possível a convivência com essa condição.

Um marco na luta pela aceitação da obesidade (ou das pessoas obesas) e, por conseguinte, do movimento fat pride, foi o lançamento do livro Fat'So? de Marilyn Wann, em 1998. Wann é uma líder do movimento fat pride nos Estados Unidos, e com o lançamento do livro e da revista com o mesmo nome, o assunto ganha projeção mundial..$^{22} \mathrm{O}$ cunho de aceitação social promovido pela vertente norte-americana do movimento traz algumas reflexões e avanços importantes, como melhor vivência da sexualidade por mulheres obesas participantes ativas do movimento. ${ }^{23}$ Outro estudo faz correlações entre os movimentos ativistas de gays e pela igualdade de gêneros com o movimento fat pride. ${ }^{22}$

No meio científico, o assunto ainda apresenta pouco destaque. Em novembro de 2012, a busca pelo unitermo "fat pride" no site do Google Acadêmico traz apenas 141 resultados.

No Brasil, o surgimento do fat pride é bem mais recente. As primeiras manifestações surgiram apenas no fim da década de 90, apresentando perfil mais "midiático", se comparado ao movimento social mais estruturado, como nos Estados Unidos. O movimento foi assunto de revistas de circulação nacional recentemente, mas com grande foco no anúncio de produtos para pessoas com excesso de peso. Recentemente começaram a acontecer eventos de moda e concursos de 
beleza para pessoas acima do peso. Um exemplo desta condição são os anúncios de moda íntima para pessoas com excesso de peso, que mostra uma erotização da publicidade não observada no modelo do movimento originalmente surgido em solo norte-americano, o que caracteriza uma diversidade importante no modo de pensar o movimento fat pride em solo brasileiro.

\section{Considerações finais}

pode-se perceber, a partir da revisão não sistemática da literatura sobre o assunto, que a obesidade constitui uma condição estigmatizante. Essa condição é fortemente sustentada pelo apelo midiático, cabendo à mídia o papel de definir um padrão de beleza e saúde longe da realidade de grande parte da população mundial, assolada pelo fenômeno de transição nutricional e que sofre as consequências do avanço dos níveis de obesidade em escala epidêmica. Os veículos midiáticos ainda associam à obesidade valores negativos, já historicamente associados à condição, mas que ganham grande realce com a veiculação de um modelo de corpo controlado por dietas rigorosas, exercícios extenuantes e uso de tecnologias de modificação corporal, além de caracterizar a obesidade como uma condição associada a valores morais e sociais negativos.

Sendo a obesidade uma condição de difícil controle, e como parte do processo de medicalização social atual, a obesidade passa por um processo de medicalização importante. A cirurgia bariátrica surge como expoente desse processo, desempenhando papel de ícone na luta contra a obesidade, por promover mudanças corporais importantes, mais efetivas que as terapias convencionalmente utilizadas nas tentativas de redução ponderal. Vale ressaltar que este crescimento da cirurgia bariátrica apóia-se fortemente em um modelo de saúde altamente reducionista, característico do paradigma biomédico, que considera apenas as mudanças corporais promovidas pela cirurgia, sem considerar os aspectos psicossociais resultantes do procedimento cirúrgico.

Aos indivíduos que batalham diariamente contra a condição da obesidade e contra os estigmas decorrentes desta condição, o movimento fat pride surge como uma tentativa de superação do estigma, desempenhando papel importante na aceitação dos indivíduos obesos pela sociedade através de ações estruturadas. O surgimento deste movimento no Brasil, entretanto, se caracteriza muito mais pelo apelo midiático, e se embasa numa tendência de criar um movimento comercial, visando atingir uma população desejosa de consumir, mas alijada dos padrões de consumo voltados para corpos magros e modelados. 


\section{Referências}

1. World Organization Health. Obesity: preventing and managing the global epidemic: report of a WHO consultation. Genebra: WHO; 2000.

2. Kac G, Sichieri R, Gigante DP. Epidemiologia nutricional. Rio de Janeiro: Atheneu; 2007.

3. Instituto Brasileiro de Geografia e Estatística. Pesquisa de Orçamentos Familiares 20082009 - Antropometria e estado nutricional de crianças, adolescentes e adultos no Brasil. Rio de Janeiro [Internet]. Rio de Janeiro: IBGE; 2010 [citado 10 jan. 2014]. Disponível em: http://www.ibge.gov.br/estadosat/temas.php?sigla=rj\&tema=pofantropometrica_2009

4. Mattos RS, Luz MT. Sobrevivendo ao estigma da gordura: um estudo socioantropológico sobre obesidade. Physis Revista de Saúde Coletiva 2009; 19(2):489-507.

5. Mattos RdS. Sou gordo, sou anormal? Arquivos em Movimento [Internet] 2007; 3(2).

6. Mendonça CP, Anjos LA. Aspectos das práticas alimentares e da atividade física como determinantes do crescimento do sobrepeso/obesidade no Brasil. Cad. Saúde Pública 2004; 20(3):698-709.

7. Brasil. Ministério da Saúde. Promovendo a alimentação saudável. Guia alimentar para a população brasileira. Brasília: Ministério da Saúde; 2006.

8. Garcia RWD. Reflexos da globalização na cultura alimentar: considerações sobre as mudanças na alimentação urbana. Rev. Nutr. 2003; 16(4):483-192.

9. Mondini L, Monteiro CA. Mudanças no padrão de alimentação. In: Monteiro CA, editor. Velhos e novos males da saúde do país. São Paulo: Núcleo de Pesquisas Epidemiológicas em Nutrição e Saúde, Universidade de São Paulo; 2000.

10. Goffman E. Estigma: Notas sobre a manipulação da identidade deteriorada. $4^{\mathrm{a}}$ ed. Rio de Janeiro: Ltc; 1988.

11. Gilman SL. Obesidade como deficiência: o caso dos judeus. Cadernos Pagu 2004; (23):329-353.

12. Boero N. All the news that's fat to print: the american "obesity epidemic" and the media. Qualitative Sociology 2007; 30(1):41-60.

13. Eknoyan G. A History of obesity, or how what was good became ugly and then bad. Adv. Chronic Kidney Dis. 2006; 13(4):421-427.

14. Miskolci R. Corpos elétricos: do assujeitamento à estética da existência. Estudos Feministas 2006; 14(3): 272-281.

15. Rasmussen N. Weight stigma, addiction, science, and the medication of fatness in mid-twentieth century America. Sociol. Health Illn 2012; 34(6):880-95.

16. Gremillion H. The cultural Politics of body size. Annual Review of Anthropology. 2005; 34:13-32.

17. Gracia-Arnaiz M. Fat bodies and thin bodies. Cultural, biomedical and market discourses on obesity. Appetite 2010; 55(2):219-25.

18. Sontag SA. A doença como metáfora. Rio de Janeiro: Graal; 1984. 
19. Rosa TV, Campos DTF. O sofrimento psíquico na condição obesa e a influência da cultura. Estudos 2008; 35(5):967-979.

20. Felippe FM. O peso social da obesidade [tese]. Porto Alegre: PUCRS; 2001.

21. Yoshino NL. A Normatização do corpo em “excesso". [tese] Campinas: Universidade Estadual de Campinas. Faculdade de Ciências Médicas Campinas. 2010: p. 377.

22. Murray S. Doing politics or selling out? living the fat body. Women's Studies: An interdisciplinary Journal 2005; 34(3-4):265-277.

23. Gailey JA. Fat shame to fat pride: fat women's sexual and dating experiences. Fat Studies: An Interdisciplinary Journal of Body Weight and Society 2012; 1(1):114-127.

24. Conrad P. The medicalization of society: on the transformation of human conditions into treatable disorders. N Engl J Med 2008; 358:2081-2082.

25. Camargo Junior KRD. Biomedicina saber e ciência: uma abordagem crítica. Hucitec; 2003.

26. Yokokura AVCP, Silva AAM, Araújo GF, Cardoso LO, Barros LCMM, Sousa SMA. Obesidade e cirurgia bariátrica no olhar dos ex-obesos mórbidos. Saúde em debate 2011; 35(90):462-9.

27. National Association to Advande Fat Acceptance. NAAFA: the National Association to Advance Fat Acceptance [Online]. [acesso em: 22 dez. 2012]. Disponível em: http://www.naafaonline.com/ $\operatorname{dev} 2 /$ "http://www.naafaonline.com/dev2/ 
